\title{
VLF Measurements and Modeling of the D-Region Response to the 2017 Total Solar Eclipse
}

\author{
Wei Xu ${ }^{\circledR}$, Robert A. Marshall ${ }^{\circledR}$, Member, IEEE, Antti Kero, Esa Turunen, \\ Douglas Drob, Jan Sojka, and Don Rice
}

\begin{abstract}
In this paper, we report measurements in Colorado and Utah of the disturbed very-low-frequency (VLF) signals from the NML Navy transmitter in North Dakota during the 2017 solar eclipse. Using an occultation mask of solar fluxes together with detailed chemistry and VLF propagation simulations, we quantify the D-region response to the eclipse, in terms of electron density variation, as well as the expected signatures of VLF transmitter signals. The VLF measurements, including an anomalous amplitude enhancement recorded in UT, can be quantitatively explained using the Wait and Spies ionospheric profile with a sharpness parameter of $\beta=0.3 \mathrm{~km}^{-1}$ above $\sim 55 \mathrm{~km}$ and an increase in the D-region ionosphere height of $\Delta h^{\prime} \simeq 8 \mathrm{~km}$. This sharpness parameter is consistent with previously reported rocket measurements and first-principles calculations. The best-fit results suggest a reduction of D-region electron density by $\sim 90 \%$ during the eclipse in the D-region, implying an occultation of Lyman- $\alpha$ by nearly $99 \%$. This finding agrees with detailed calculations of time-dependent obscuration factors utilizing the He 30.4-nm images from Solar Dynamics Observatory as a proxy for the distribution of Lyman- $\alpha$ across the solar disk and limb. Moreover, the present results show that subionospheric VLF propagation is sensitive to the sharpness parameter of the electron density profile in the D-region. Previously reported first-principles simulations have shown that the sharpness parameter is mostly controlled by the background concentration of minor neutral species. Thus, the VLF technique can be likely used to remotely sense these neutral species at and below the effective reflection altitudes of VLF waves.
\end{abstract}

Index Terms-D-region ionosphere, earth-ionosphere waveguide, finite-difference frequency domain (FDFD), ionosphere, ionosphere chemistry, Lyman- $\alpha$ radiation, solar eclipse, subionospheric very-low-frequency (VLF) remote sensing, transmitter, VLF.

Manuscript received December 21, 2018; revised March 25, 2019; accepted March 28, 2019. The works of W. Xu and R. A. Marshall were supported by the National Aeronautics and Space Administration (NASA) under Grant NNX17AI05G. The work of A. Kero was supported in part by the Tenure Track Project in Radio Science at Sodankylä Geophysical Observatory and in part by the ISSI Project: Negative Ions in the Solar System. The work of D. Drob was supported by NASA through the U.S. Naval Research Laboratory under Grant NNH17AE63I. This work was supported by NSF through Utah State University under Grant AGS-0962544. (Corresponding author: Wei Xu.)

W. Xu and R. A. Marshall are with the Department of Aerospace Engineering Sciences, University of Colorado Boulder, Boulder, CO 80309 USA (e-mail: wei-xu@colorado.edu; robert.marshall@colorado.edu).

A. Kero and E. Turunen are with the Sodankylä Geophysical Observatory, University of Oulu, 90014 Oulu, Finland.

D. Drob is with the Space Science Division, Naval Research Laboratory, Washington, DC 20375 USA.

J. Sojka and D. Rice are with the Center for Atmospheric and Space Sciences, Utah State University, Logan, UT 84322 USA.

Color versions of one or more of the figures in this paper are available online at http://ieeexplore.ieee.org.

Digital Object Identifier 10.1109/TGRS.2019.2914920

\section{INTRODUCTION}

$\mathbf{T}$ HE D-region of the ionosphere between $60-$ and $90-\mathrm{km}$ altitude is integrally important in a number of areas of heliophysics, aeronomy, and long-range communications. It couples to the higher regions of the ionosphere and to the neutral atmosphere through atmospheric gravity waves [1], energetic particle precipitation, and transport [2]. Energetic particles precipitating from the radiation belts, a region of space near the earth (approximately from 1.2 to 7 earth radii) with relatively high-energy charged particles, can deposit their energy in the D-region, where ion chemistry determines the production of odd nitrogen and its descent to lower altitudes [3], [4]. The D-region also controls the propagation of very-low-frequency (VLF) waves, with frequencies of 3-30 kHz, used for long-range communications [5]. Moreover, the D-region dynamics is crucial for the coupling of terrestrial VLF waves (for example, from atmospheric lightning discharges) to the outer ionosphere [6].

Despite the above-mentioned importance, the D-region ionosphere is particularly difficult to measure due to the very low electron densities. High-power incoherent scatter radars (ISRs) have been recently employed to investigate the D-region [7], but only during periods of enhanced ionization and using long integration times. Riometers measure HF absorption through the D-region [8] but can only infer the electron density as a single pierce point. The most sensitive method to measure the D-region is using VLF subionospheric remote sensing (SRS). This technique is highly sensitive to D-region fluctuations; VLF frequencies can propagate in the earth-ionosphere waveguide over long distances with low losses ( $\sim 2 \mathrm{~dB}$ per megameters); the received VLF signals are modulated by the D-region ionosphere and thus carry direct information about D-region variability [9]. Moreover, it has been recently suggested that the group velocity of extremely low-frequency (ELF) waves has a particular sensitivity to the sharpness of electron density profile in the D-region [10].

Solar eclipses have historically provided insightful information about the D-region characteristics and ionization sources [11]-[15]. Using rocket experiments, Sears et al. [13] directly investigated the variation of electron density in the D-region ionosphere during the 1966 solar eclipse in South America. However, previous studies of the solar eclipse using rocket sounding mostly measured the electron density at alti-

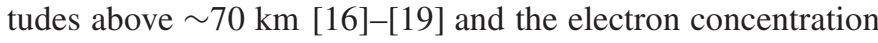
below this altitude remains poorly investigated. More recently, 
a detailed modeling study of the solar eclipse in Europe on August 11, 1999 [14] revealed that the amplitude of VLF signals was usually enhanced for relatively short propagation paths $(<2000 \mathrm{~km})$ and diminished for relatively long propagation paths $(>10000 \mathrm{~km})$. In contrast, our VLF observation in UT during the 2017 eclipse exhibited distinctive characteristics: for a short propagation path, the VLF amplitude first decreased by $\sim 7.5 \mathrm{~dB}$ as the eclipse moved toward the path and was then temporarily enhanced by $\sim 1.5 \mathrm{~dB}$ at the onset of the eclipse. Moreover, this anomalous enhancement was correlated with a C-class solar flare event that occurred near the time of totality. To explain this abnormal VLF signature associated with both solar flare and eclipse events represents a major goal of this paper.

The 2017 solar eclipse provided a unique opportunity to study the D-region electron density and to validate atmospheric chemistry models. With both satellite and VLF measurements, the eclipse provided a controlled experiment in that the solar input into the ionosphere was measured by space-borne instruments, while the outcome of the ionospheric disturbance was measured by ground-based receivers. In this paper, VLF measurements and satellite data of solar fluxes are used, together with numerical simulations of the D-region chemistry and VLF propagation, to quantify the D-region response to the eclipse. The abnormal VLF measurements in UT can be explained without taking the effects of solar flare event into account. Moreover, by comparing eclipse-modulated VLF signals with modeling results obtained using different ionospheric profiles, this paper explores the D-region electron density at relatively low altitudes, which is inadequately investigated by previous rocket measurements during a solar eclipse.

\section{EXPERIMENTAL SETUP}

The total solar eclipse began at 16:48:32 UT over the Pacific and ended at 20:01:25 UT in the mid-Atlantic ocean, traversing over the Continental United States from Oregon to South Carolina on August 21, 2017. Two VLF receivers located at Table Mountain, Colorado (TMC) $\left(39.76^{\circ} \mathrm{N}, 105.20^{\circ} \mathrm{W}\right)$ and Bear Lake, Utah (BLU) $\left(42^{\circ} \mathrm{N}, 111.33^{\circ} \mathrm{W}\right)$ monitored the amplitude and phase of the signal from the Navy VLF transmitter NML in Lamoure, North Dakota $\left(46.37^{\circ} \mathrm{N}\right.$, $98.34^{\circ} \mathrm{W}$ ), which transmits at $25.2 \mathrm{kHz}$ with a radiated power of $500 \mathrm{~kW}$.

Fig. 1 shows the totality path of the solar eclipse, as well as the great circle paths for the experiment configuration between NML and the VLF receivers in CO and UT (denoted as the NML-CO and NML-UT paths hereafter). The black lines show the path of maximum totality (solid) and the edges of totality (dashed). The VLF receivers were developed based on the AWESOME receiver system [20], [21]; the two receiver locations were well suited for the eclipse observation; both NML-CO ( 921 km) and NML-UT $(\sim 1145 \mathrm{~km})$ are short compared to typical paths used for VLF remote sensing.

Also shown is the fraction of obscuration at $75-\mathrm{km}$ altitude for the far-/mid-ultraviolet (FUV/MUV) and extreme ultraviolet (EUV) wavelength range at 17:50 UT, which is calculated
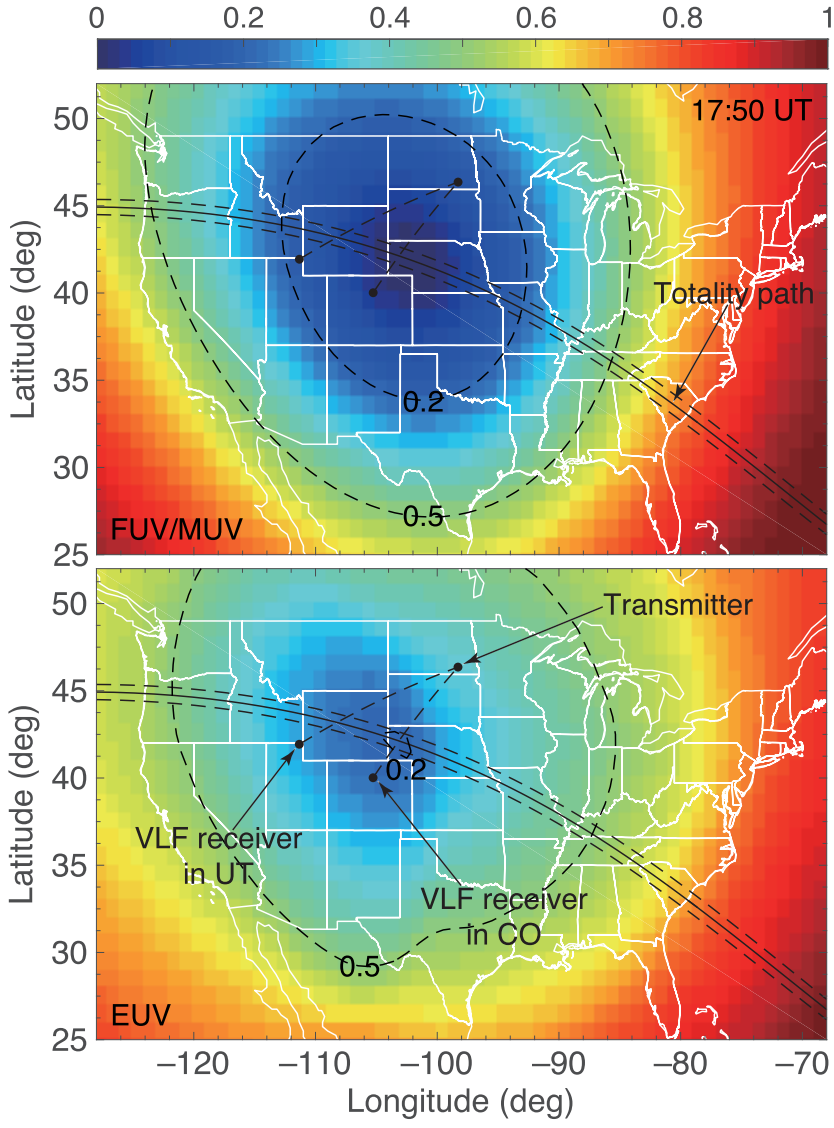

Fig. 1. Fraction of solar radiation at $75-\mathrm{km}$ altitude in the (Top) FUV/MUV and (Bottom) EUV wavelength range reaching the earth's atmosphere at 17:50 UT on August 21, 2017 over the continental United States. The dots mark the VLF transmitter (NML) in Lamoure, ND and the VLF receivers installed near TMC and BLU.

utilizing the methodology described in [22]-[24] based on earlier work of Marriott et al. [25]. This methodology is based on the measurements by the Solar Dynamics Observatory (SDO) Atmospheric Imaging Assembly (AIA) [26] during the eclipse. Specifically, the occultation factors were computed from high-resolution SDO AIA images of the solar disk using the Naval Observatory Vector Astrometry Software [27]. These factors, with values between 0 and 1, describe the reduction in solar radiation caused by the eclipse. For the simulations presented here, these factors are calculated over the simulation domain at 1 -min cadence with $1^{\circ}$ resolution in latitude and longitude.

The obscuration of EUV and FUV/MUV sources is different: the FUV/MUV wavelengths are blocked over a notably larger area. In the totality region, solar photons in the FUV/MUV range can be fully obstructed by the moon's shadow, whereas the maximum reduction in EUV photons is $\sim 80 \%$. The noncircular patterns for the occultation factors in the EUV range are caused by the nonuniform distribution of EUV fluxes across the solar disk as a result of active regions and corona structure.

\section{MOdEL FORMULATION}

To understand the eclipse-induced effects, we begin by forward-modeling the D-region and VLF responses to solar 

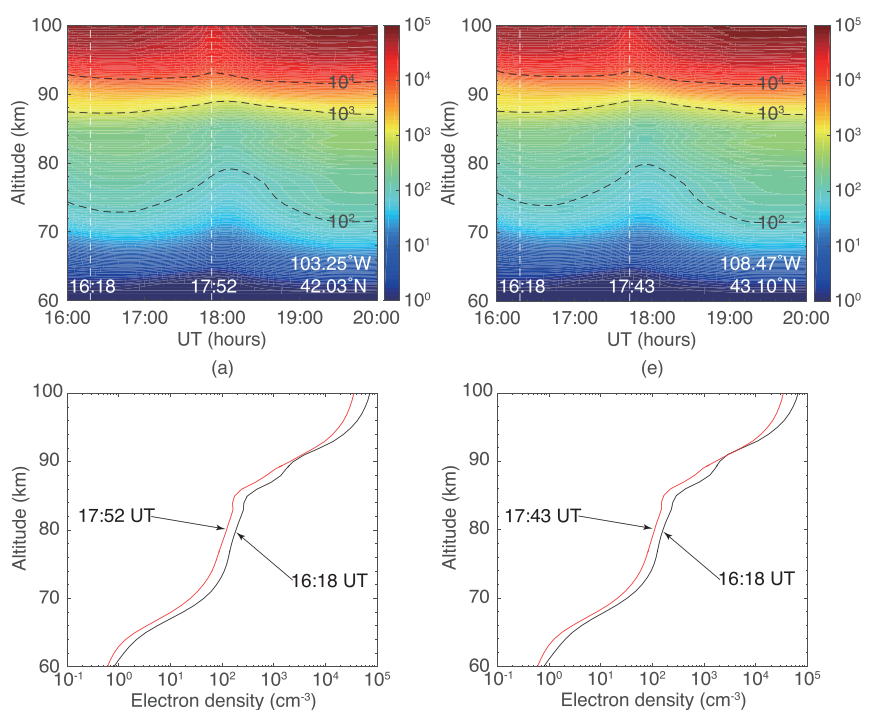

(b)

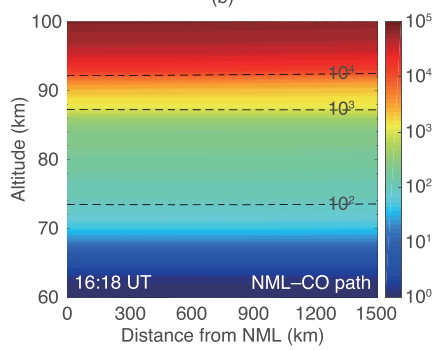

(c)

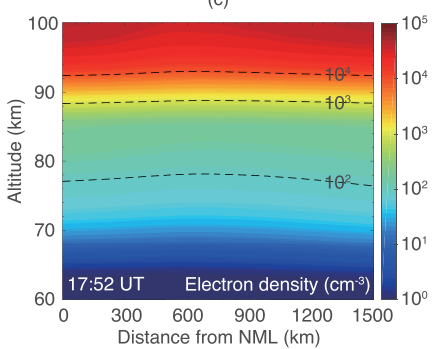

(d)

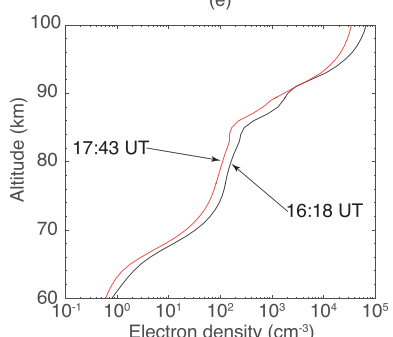

(f)

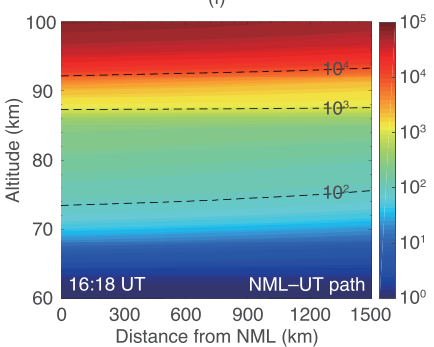

(g)

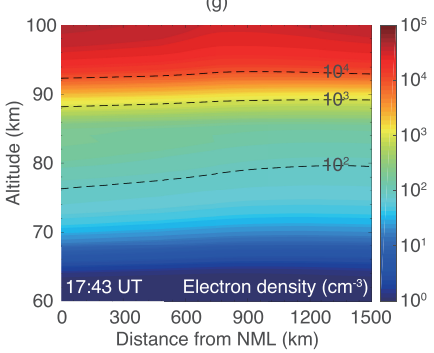

(h)

Fig. 2. SIC modeling results of (a) altitude profiles of electron density at $42.03^{\circ} \mathrm{N}, 103.25^{\circ} \mathrm{W}$ between 16:00 and 20:00 UT on August 21, 2017. (b) Electron density versus altitude at 16:18 and 17:52 UT. SIC modeling results of 2-D (range, altitude) slices of electron density profiles at (c) 16:18 UT and (d) 17:52 UT along the NML-CO path. These results are obtained by considering that the occultation of Lyman- $\alpha$ follows that of EUV sources (SIC-EUV profiles). (e)-(h) Similar results, but for the NML-UT path.

eclipse using the occultation factors and first-principles models. These simulations were performed in order to examine: 1) the dependence of D-region electron density on the occultation of solar inputs and 2) if the VLF measurements can be explained through first-principles modeling. However, the occultation mask utilized here only describes the solar obscuration as two wavelength channels and the occultation data of Lyman- $\alpha$ radiation, which is the major ionization source of D-region [28], is not explicitly available. To resolve this uncertainty, we have also quantified the ionosphere response by finding the D-region parameters that best fit the VLF measurements using the Wait and Spies (WS) ionospheric profile [29]. The goal is to: 1) compare the electron concentration in the D-region; 2) inversely estimate the D-region variation and Lyman- $\alpha$ occultation; and 3) check if the derived Lyman- $\alpha$ occultation is consistent with the occultation mask and/or satellite measurements.

In the forward simulation, two numerical models are employed, including the Sodankylä ion and neutral chemistry (SIC) model [30] and a finite-difference, time-domain (FDTD) model [31]. First, we incorporate solar occultation factors into the SIC model. Second, a sequence of 2-D (range, altitude) slices of the electron density profiles between the transmitter and VLF receivers are extracted from the chemistry modeling results; 30 locations were uniformly chosen along each transmitter-receiver path, up to a distance of $1500 \mathrm{~km}$ away from the transmitter. Finally, these profiles are used as the input to the FDTD VLF propagation model to simulate the expected VLF amplitude and phase at the receivers. As the eclipse moves across the country, in order to capture the temporal evolution, the electron density profiles are updated at 1-min time intervals and the VLF propagation model is rerun with updated inputs.

The occultation mask provides the eclipse-induced obscuration for solar input in two wavelength regimes at the time when forward simulations were carried out. As such, this model leaves out important wavelength-dependent characteristics of solar fluxes, particularly Lyman- $\alpha$ emission (1214 $\AA$ ). In this mask, Lyman- $\alpha$ falls under the EUV but, in reality, originates primarily from the chromosphere and transition region of the solar atmosphere [32]. It is also inaccurate to use the average value of EUV and FUV/MUV occultation factors because Lyman- $\alpha$ radiation is neither limb brightening nor darkening.

Because of this uncertainty, we have calculated the ionospheric profiles for two scenarios: the occultation of Lyman- $\alpha$ follows that of solar fluxes either in the EUV or FUV/MUV wavelength range (denoted as the SIC-EUV and SIC-FUV profile hereafter). In reality, the occultation of Lyman- $\alpha$ during the eclipse lies between these two limiting cases. Therefore, they provide reasonable bounds on the eclipse-induced effects in D-region ionosphere. When comparing the derived solar occultation with satellite measurements [Fig. 7(d)], occultation factors are calculated separately using the images of He 30.4-nm emissions as an approximate guess for Lyman- $\alpha$. We emphasize that these factors are well between the EUV and FUV/MUV factors and this scenario is also captured by the SIC-EUV and SIC-FUV profiles [see Fig. 7(c) and (d)]. As explained later in this paper, using these factors as the obscuration of Lyman- $\alpha$ in chemistry simulations cannot reproduce our VLF measurements because of the steepness of ionospheric profiles at altitudes between $\sim 55$ and $\sim 72 \mathrm{~km}$.

The SIC model is a 1-D atmospheric chemistry model that dynamically solves for the concentration of 16 minor neutral species and 72 ionic species at altitudes between 20 and $150 \mathrm{~km}$ with $1-\mathrm{km}$ resolution. This model has been widely used to simulate the atmospheric chemistry changes induced by energetic particle precipitation [30], [33], [34]. Vertical transport is included through molecular and eddy diffusion, neglecting transport by prevailing neutral wind. The latest version of this model takes into account 389 ionneutral and neutral-neutral reactions and 2523 ion-ion and 
electron-ion recombination reactions; the negative ion chemistry considered in the SIC model is identical to the Whole Atmosphere Community Climate Model with D-region ion chemistry (WACCM-D) [33]. SIC is mainly driven by solar radiation and the background profile of the neutral density is obtained from the NRLMSISE-00 model [35] using the daily average values of solar radio flux $\left(F_{10.7}\right)$ and the geomagnetic activity index $\left(A_{\mathrm{p}}\right)$. More details about the SIC model can be found in [30] and [36].

The FDTD model [31] was modified for long-range VLF propagation by Marshall et al. [37], which calculates the electromagnetic fields in the time-domain from Maxwell's equations, and the currents in the ionosphere using the Langevin equation. This model can simulate the propagation of frequencies from $\sim 100 \mathrm{~Hz}$ up to $500 \mathrm{kHz}$ and can include arbitrary ground conductivity, ionospheric density, atmospheric density, and the earth's magnetic field. The FDTD model extracts the amplitude and phase along the ground at discrete frequencies by calculating cumulative discrete Fourier transforms at specified grid locations. It has been validated through a direct comparison with the long wave propagation capability (LWPC) code [38], both in [37] and in simulations for this paper. Using identical ionosphere and ground parameters, FDTD results show very good agreements with the LWPC code; the maximum amplitude difference is approximately $1 \mathrm{~dB}$ with a phase difference typically less than a few degrees, for different simulation paths and transmitter frequencies [37].

\section{RESUlts}

\section{A. SIC Ionospheric Profile}

SIC-EUV and SIC-FUV ionospheric profiles are presented in Figs. 2 and 3, respectively. Fig. 2(a) shows the electron density profile calculated by the SIC model at $42.03^{\circ} \mathrm{N}$, $103.25^{\circ} \mathrm{W}$ between 16:00 and 20:00 UT. Fig. 2(b) shows the altitude profile of electron density at 16:18 and 17:52 UT, corresponding to the white dashed lines denoted in Fig. 2(a). Fig. 2(c) and (d) shows 2-D (range, altitude) slices of the electron density along the NML-CO path at 16:18 and 17:52 UT, respectively. Fig. 2(e)-(h) shows the similar results, but for the NML-UT path.

Due to a larger obscuration of solar FUV, the variation of D-region electron density is more pronounced in the SIC-FUV profile. For both the transmitter-receiver paths, the altitude at which the density equals to $10^{2}$ electrons $/ \mathrm{cm}^{3}$ is increased by approximately $5 \mathrm{~km}$ during the eclipse in the SIC-EUV profiles [Fig. 2(a) and (e)], whereas the increase is approximately $11 \mathrm{~km}$ in the SIC-FUV profiles [Fig. 3(a) and (e)]. Moreover, the electron density at the reflection altitudes of VLF signals during the totality is reduced by up to $\sim 45 \%$ and $\sim 71 \%$ in the SIC-EUV [Fig. 2(b) and (f)] and SIC-FUV [Fig. 3(b) and (f)] profiles, respectively.

VLF amplitude and phase data recorded by the receiver located at TMC, are presented in Fig. 4(a), while measurements near BLU, are presented in Fig. 4(b). The VLF amplitude in $\mathrm{CO}$ increased by $\sim 10 \mathrm{~dB}$ while the phase decreased by $\sim 140^{\circ}$. Conversely, VLF measurements in BLU showed an amplitude drop of $\sim 7.5 \mathrm{~dB}$ followed by a temporary
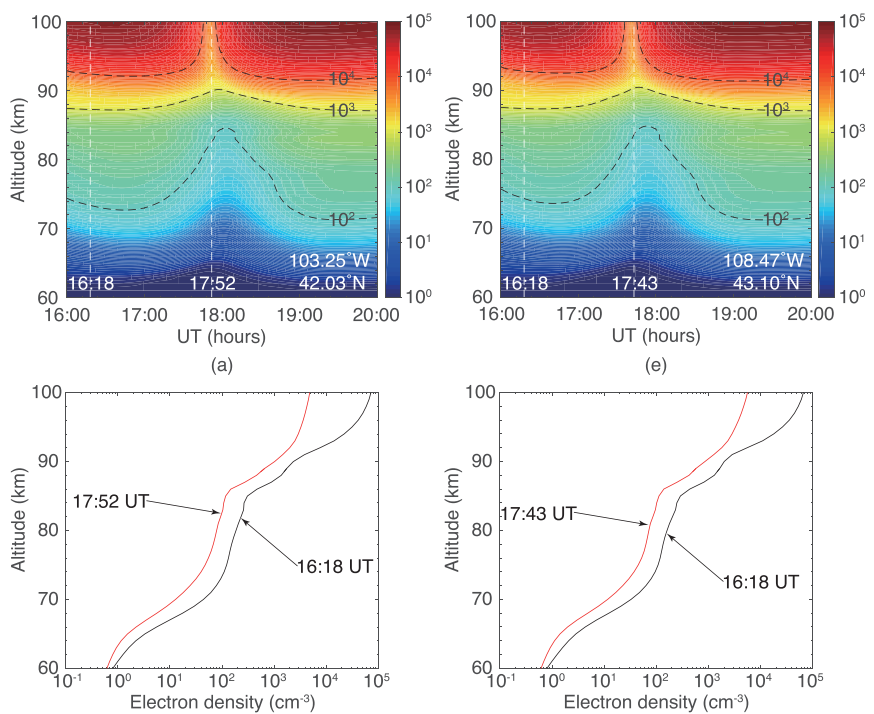

(b)

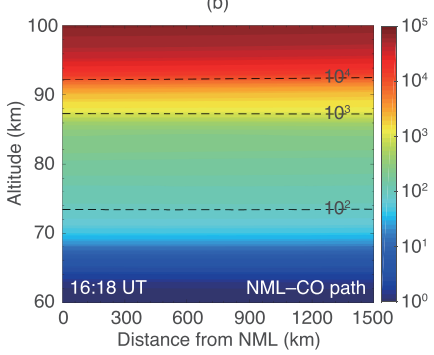

(c)

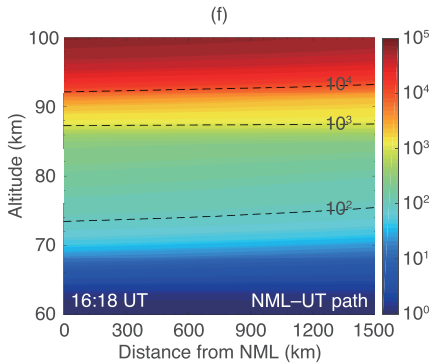

(g)

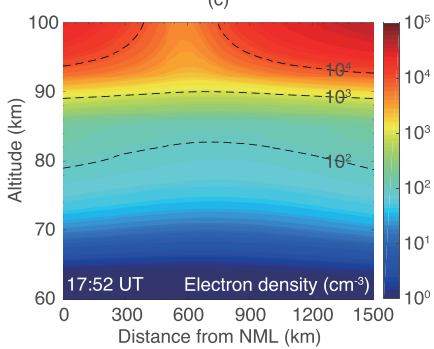

(d)

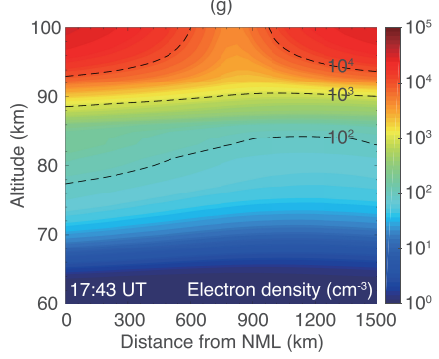

(h)

Fig. 3. (a)-(h) Similar to Fig. 2, but obtained by considering that the occultation of Lyman- $\alpha$ follows that of FUV/MUV sources (SIC-FUV profiles).

enhancement of $\sim 1.5 \mathrm{~dB}$ during the eclipse. The phase change was approximately $-100^{\circ}$.

The VLF amplitude and phase calculated using FDTD simulations are also shown in Fig. 4 as dashed lines. These results are obtained by propagating VLF signals through either SIC-EUV or SIC-FUV profiles, using the FDTD model with a spatial resolution of $1 \mathrm{~km}$ and a temporal resolution of $1 \mathrm{~min}$. For the $\mathrm{CO}$ site, the simulated amplitude change is -0.6 and $-1.3 \mathrm{~dB}$ for the SIC-EUV and SIC-FUV profiles, respectively. The corresponding phase change is $-29^{\circ}$ and $-47^{\circ}$. For the BLU site, the simulated amplitude change is +1.7 and $+2.7 \mathrm{~dB}$ for the SIC-EUV and SIC-FUV profiles, respectively. The corresponding phase change is $-27^{\circ}$ and $-50^{\circ}$. It is clear that these SIC-estimated ionospheric profiles do not reproduce the observed VLF response. The main reason, as will be explained later, is that the default profiles of minor neutral species in the SIC model cannot produce sufficient electron density at altitudes between 55 and $72 \mathrm{~km}$. 


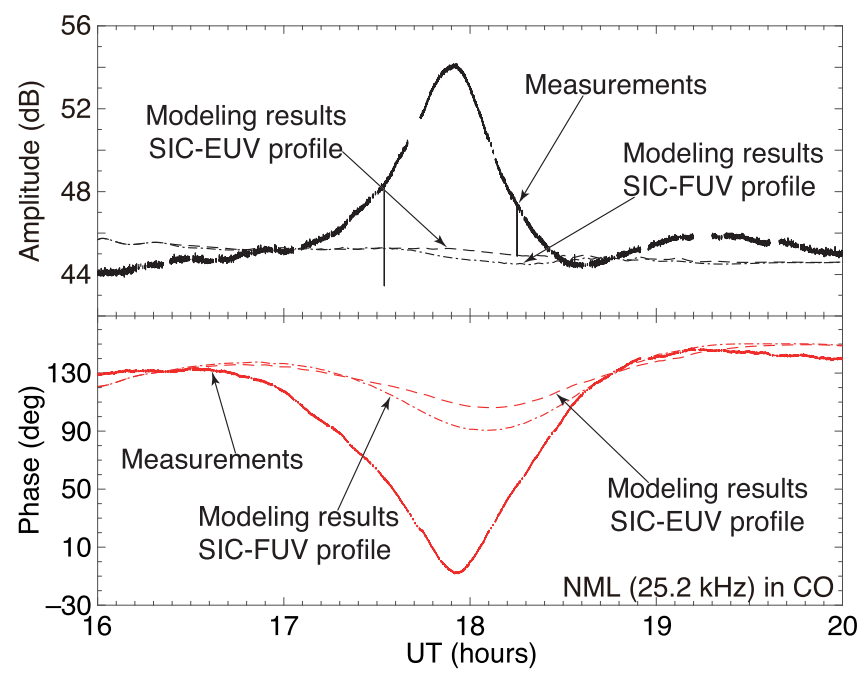

(a)

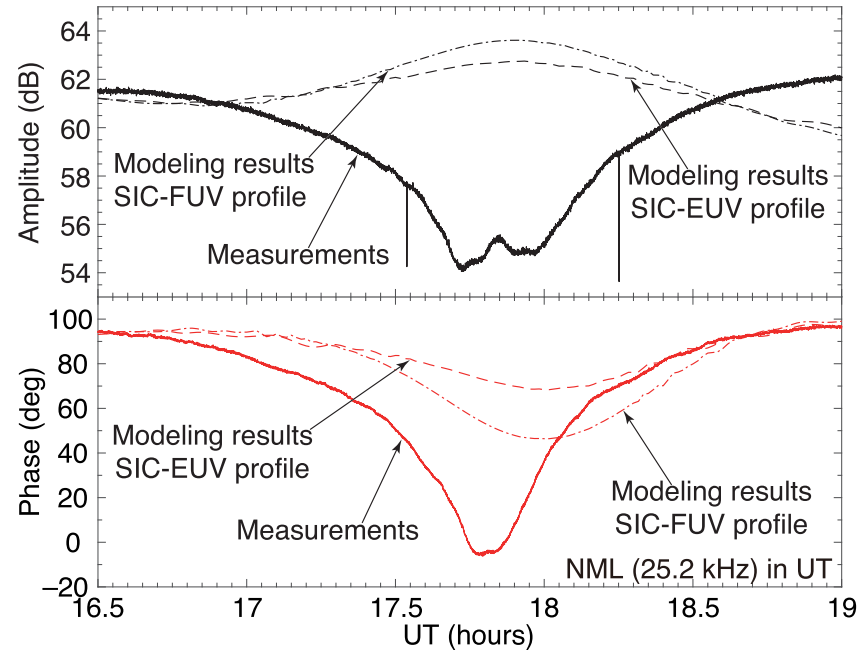

(b)

Fig. 4. (a) Comparison of VLF (Top) amplitude and (Bottom) phase between observational data (solid line) recorded near TMC on August 21, 2017 and modeling results (dashed lines). (b) Similar results, but for the VLF measurements near BLU.

\section{B. Ionospheric Profile Fitting}

To explain the observed VLF signals, as motivated by previous studies [14], [15], we employ the two-parameter WS profile [29]. In this profile, the ionosphere electron density $n_{\mathrm{e}}$ [electrons $/ \mathrm{m}^{3}$ ] at altitude $h[\mathrm{~km}$ ] is defined by a characteristic height $h^{\prime}[\mathrm{km}]$ and sharpness parameter $\beta\left[\mathrm{km}^{-1}\right]$

$$
n_{\mathrm{e}}(h)=1.43 \times 10^{13} e^{-0.15 h^{\prime}} e^{(\beta-0.15)\left(h-h^{\prime}\right)} .
$$

In this parametric study, the WS ionosphere and FDTD model are utilized in an optimization algorithm in order to explain the VLF measurements, with three constraints. First, $\beta$ is assumed to be invariant throughout the eclipse. This is motivated by the SIC results presented in Figs. 2 and 3, showing that the vertical structure of the density profile is almost unchanged throughout the eclipse in both SIC-EUV and SIC-FUV profiles. Second, the ionosphere is assumed to be homogeneous along the transmitter-receiver path; the
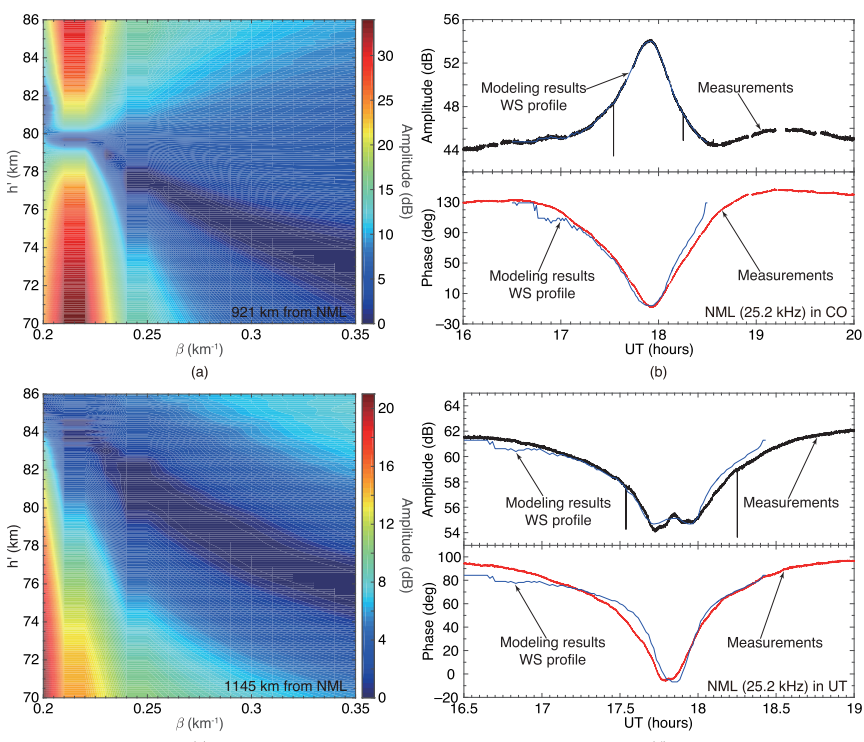

Fig. 5. FDTD modeling results of VLF amplitude changes calculated using the WS profile with arbitrary combinations of $h^{\prime}$ and $\beta$ for two locations. (a) $921 \mathrm{~km}$ and (c) $1145 \mathrm{~km}$ away from NML. (b) Comparison of VLF (Top) amplitude and (Bottom) phase between eclipse measurements in $\mathrm{CO}$ and the best fit obtained using the WS profile. (d) Similar results, but for the best fit to the VLF measurements in BLU.

electron density profile at any location along the path follows the same WS formula. This assumption is adopted because we focus on quantifying the average eclipse-induced effects on the propagation path, which allow direct comparison with satellite observation of solar input reduction. It has been verified through simulations of lightning sferics that VLF propagation is mostly controlled by the path-average ionospheric height and sharpness, even in the presence of significant inhomogeneities in these parameters [39]. We emphasize that varying the ionospheric height and sharpness along the transmitter-receiver path can precisely reproduce the measured VLF signals, but then this optimization problem is loosely constrained and multiple solutions can be obtained for our VLF measurements.

Finally, to limit the degrees of freedom in this optimization problem, the relative variation of characteristic height during the eclipse, i.e., relative change in $h^{\prime}$ versus time $t: \Delta h^{\prime}(t)=$ $h^{\prime}(t)-h^{\prime}(t=0)$, is assumed to be the same for the two propagation paths. This assumption is valid considering that the solar obscuration along these two paths is comparable (see Fig. 1), and thus, the corresponding evolution of electron density in the D-region, which is related to $\Delta h^{\prime}(t)$ in WS profiles, should be similar. The initial value $h^{\prime}(t=0)$ is not necessarily the same because the undisturbed electron density before the eclipse was almost certainly different to some degrees for the two paths. Nevertheless, in reality, these two paths were not simultaneously influenced by the solar eclipse, and this assumption introduces a time shift in the simulated VLF signals. For the sake of direct comparison, we have corrected this effect [Figs. 5(d) and 7(a)] using the time difference in terms of maximum eclipse effects between the two paths $(\sim 4 \mathrm{~min})$. 
For the sake of completeness, we calculate the VLF amplitude and phase changes for all possible combinations of $h^{\prime}$ and $\beta$. We vary $\beta$ between 0.2 and $0.35 \mathrm{~km}^{-1}$ (step: $0.01 \mathrm{~km}^{-1}$ ) and $h^{\prime}$ between 70 and $86 \mathrm{~km}$ (step: $0.1 \mathrm{~km}$ ). The ionosphere fitting procedure is specifically as follows. First, for each $\beta$ value, we curve fit the amplitude measurements in CO using FDTD results corresponding to different $h^{\prime}$ values [Fig. 5(a)]. To imitate the day-night transition, we consider that $h^{\prime}$ increases and decreases monotonically before and after the onset of the solar eclipse, respectively. A set of $h^{\prime}$ values $\left(h^{\prime}(t)\right)$ and $\Delta h^{\prime}(t)$ is then found for each $\beta$. Next, for each $\beta$ value, the VLF amplitude measured in BLU is fitted using $\Delta h^{\prime}(t)$ obtained in the previous step for CO measurements, but with different initial values $\left[h^{\prime}(t=0)\right]$. We evaluate the goodness of curve fitting using the least-squares criterion. Finally, among all the $h^{\prime}$ and $\beta$ combinations, the sequence of ionospheric profiles $\left[\beta\right.$ and $\left.\Delta h^{\prime}(t)\right]$ that best explain the observational data in $\mathrm{CO}$ and UT are determined.

Fig. 5(a) shows the FDTD results of VLF amplitude changes calculated using the WS profile with arbitrary combinations of $h^{\prime}$ and $\beta$ for a location that is $921 \mathrm{~km}$ away from NML, representing the VLF receiver installed in CO. Similar results for the BLU receiver are shown in Fig. 5(c). These amplitude results are shifted based on the minimum value of each $\beta$ so that the lowest amplitude change in these plots is $0 \mathrm{~dB}$. Among these $h^{\prime}$ and $\beta$ combinations, the best fits to VLF amplitude measurements in CO and UT are shown in Fig. 5(b) and (d) (Top), respectively. Corresponding phase changes in $\mathrm{CO}$ and UT calculated using the best set of WS profiles are shown in Fig. 5(b) and (d) (Bottom), respectively.

Using a single set of ionospheric sharpness and $\Delta h^{\prime}(t)$, our measurements of VLF amplitude and phase in both CO and UT can be excellently explained. These profiles have the same $\beta$ value of $0.3 \mathrm{~km}^{-1}$, but $h^{\prime}$ increases from $\sim 75 \mathrm{~km}$ to $\sim 83 \mathrm{~km}$ and from $\sim 71 \mathrm{~km}$ to $\sim 79 \mathrm{~km}$ for the NML-CO and NML-UT paths, respectively. The change in $h^{\prime}$ is shown separately in Fig. 7(a). More interestingly, the anomalous amplitude measurements in UT, including the $7.5-\mathrm{dB}$ drop from 16:30 to 17:42 UT and the abnormal enhancement at the onset of the eclipse, are also quantitatively captured by this set of profiles. Of note, no flare-induced ionization is included in the present modeling. The good agreements shown in Fig. 5(d) indicate that the anomalous enhancement measured in UT was a result of waveguide propagation effects but not induced by the solar flare. This finding is not unreasonable considering that the NML-CO and NML-UT paths are close to each other so we would not expect only one of the paths to be affected by the solar flare event.

To better understand the abnormal VLF signature, we have plotted the temporal evolution of VLF amplitude versus the distance from NML, as shown in Fig. 6. Fig. 6(a) shows the VLF amplitude change calculated using the set of best-fit WS profiles for the NML-CO path before (16:30 UT), during (17:43, 17:51, and 17:59 UT) and after (18:24 UT) the solar eclipse. Similar results for the NML-UT path are presented in Fig. 6(c). Fig. 6(b) and (d) shows the zoomed-in view of the amplitude change near the TMC and BLU stations, respectively. Both VLF receivers were likely located close to
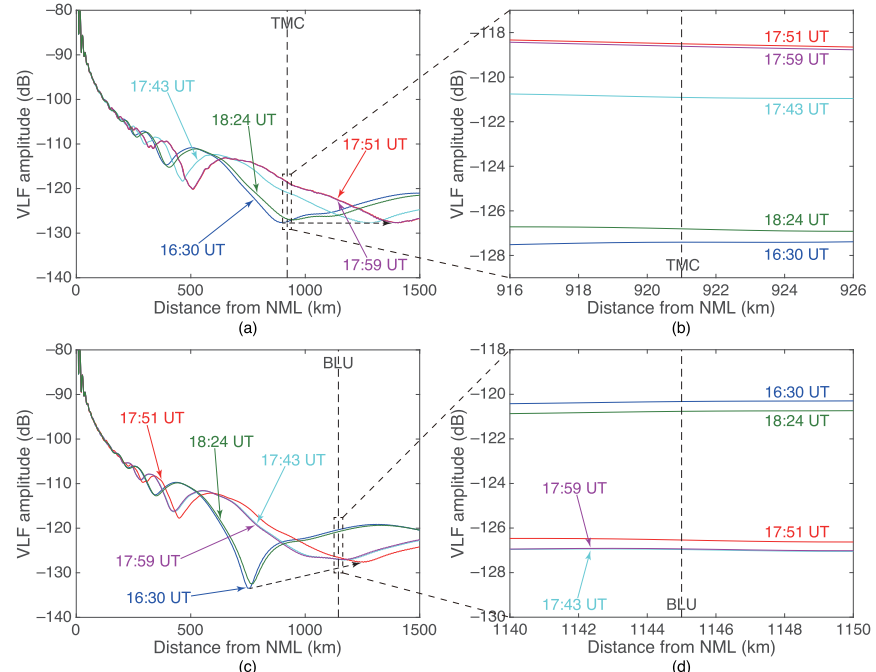

Fig. 6. Temporal evolution of VLF amplitude versus the distance from NML between 16:30 and 18:24 UT calculated using the set of best fit WS profiles obtained for the (a) NML-CO and (c) NML-UT paths. Zoomed-in view of the amplitude change near (c) TMC and (d) BLU station. The vertical dashed lines denote the location of VLF receivers.

an amplitude null as caused by the interference of propagating modes, for example, two modes with similar amplitudes and a phase difference of $180^{\circ}$ that cancel each other. Due to the reduction of solar input and variation of D-region electron density, VLF waves were reflected at considerably higher altitudes during the eclipse maxima. The amplitude null was shifted back and forth along the transmitter-receiver path, as indicated by the dashed arrows in Fig. 6(a) and (c). Consequently, the VLF amplitude response became highly nonlinear and complicated. Nevertheless, both the large enhancement measured at TMC and the nonmonotonic response measured at BLU can be fully captured by the best-fit WS profiles as clearly shown in Fig. 6(b) and (d), respectively.

The temporal evolution of the D-region electron density that best explains the VLF measurements in $\mathrm{CO}$ is shown in Fig. 7(b). As a zero-order steady-state approximation, the electron concentration in the D-region is roughly proportional to the square root of the intensity of Lyman- $\alpha$ source [30]. Using this relation, we can estimate the occultation factor for Lyman- $\alpha$ radiation during the eclipse. Fig. 7(c) shows the comparison between the derived occultation and the average value of EUV and FUV/MUV occultation factors in the totality region along the NML-CO path (see Fig. 1), while Fig. 7(d) shows the comparison with the observations by the SDO AIA. Unlike the EUV and FUV/MUV reported in previous works that account for solar limb brightening and solar limb darkening, respectively, the factors presented in Fig. 7(d) are calculated as an approximation for Lyman- $\alpha$ utilizing the SDO AIA 30.4-nm images since these two emissions have similar optical depth properties across the solar disk.

Fig. 7(e) shows the comparison of an altitude profile of electron density measured by rocket at Wallops Island, Virginia in September 1965 [40], a characteristic ionospheric profile calculated using first-principles models [41], SIC-EUV 

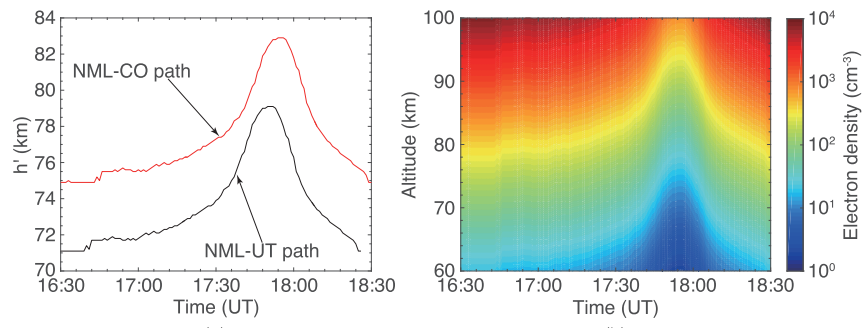

(a)

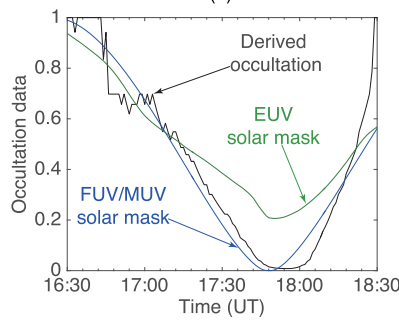

(c)

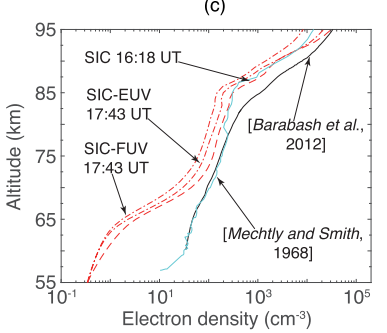

(e)

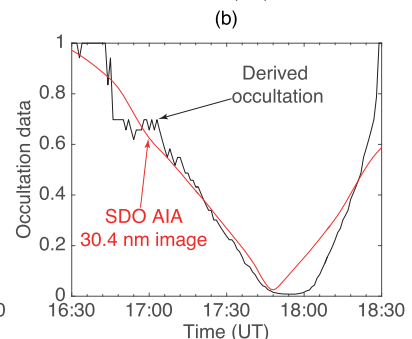

(d)

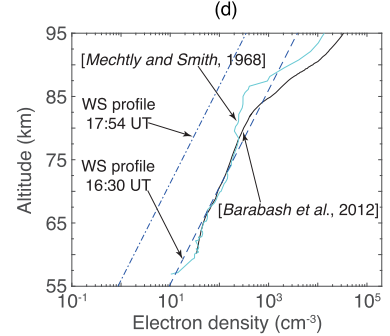

Fig. 7. Temporal evolution of (a) characteristic height for both NML-CO and NML-UT paths and (b) D-region electron density that best explains the VLF amplitude measurements in CO. (c) Comparison between the average occultation factors along the NML-CO path derived from the set of best-fitting WS profiles and those of EUV and FUV/MUV in the totality region defined in the solar mask. (d) Similar comparison, but with the average occultation factors in the totality region (NML-CO path) derived from the image of He 30.4-nm emission captured by SDO AIA. (e) Comparison of D-region electron density between rocket measurements at Wallops Island, Virginia in September 1965 [40], a characteristic ionospheric profile documented in [41], and the SIC-EUV and SIC-FUV profiles at $42.03^{\circ} \mathrm{N}, 103.25^{\circ} \mathrm{W}(16: 18$ and 17:43 UT). (f) Similar comparison, but with the best-fit WS profiles obtained for the NML-CO path.

and SIC-FUV profiles at $42.03^{\circ} \mathrm{N}, 103.25^{\circ} \mathrm{W}(16: 18$ and 17:43 UT). Fig. 7(f) shows a similar comparison, but with the WS profiles along the NML-CO path (16:30 and 17:54 UT). Note that, at 16:18 UT, the SIC-EUV profile is the same as SIC-FUV.

As shown in Fig. 7(a), the eclipse-induced D-region variation can be briefly summarized as that the effective reflection altitude increased by $\sim 8 \mathrm{~km}$, approximately from 71 to $79 \mathrm{~km}$ for the NML-UT path and from 75 to $83 \mathrm{~km}$ for the NML-CO path. Moreover, this change in $h^{\prime}$ indicates a reduction of up to $\sim 90 \%$ in electron density at the reflection altitudes of VLF signals. To explain this variation of electron density, a reduction of nearly $99 \%$ in solar Lyman- $\alpha$ is required in the chemistry simulations, significantly more than that predicted by the EUV occultation, but close to FUV/MUV. A reasonable proxy for the true obscuration of Lyman- $\alpha$ taking into account the variation due to active regions in the solar disk is the He 30.4-nm emission line. As evidenced in Fig. 7(d), the derived occultation agrees with that calculated using the SDO AIA image of 30.4-nm emission, especially the falling

part. We emphasize that the derived occultation is obtained using a zero-order approximation that the D-region electron density is roughly proportional to the square root of the intensity of solar Lyman- $\alpha$. However, the electron recombination processes and ion chemistry are not considered in this calculation; in reality, the D-region chemistry is significantly more complicated than this simplification. The time difference between the occultation factors defined in the solar mask and those derived using VLF data is mainly due to D-region chemistry.

\section{Difference Between SIC and WS Profiles}

The set of WS profiles that explain VLF measurements is different from SIC results in mainly two aspects, as shown in Fig. 7(e) and (f). First, the reduction of the D-region electron density derived from the WS profiles is more dramatic than the SIC profiles. More importantly, the WS profiles have the same sharpness parameter of $0.3 \mathrm{~km}^{-1}$ at the reflection altitudes of VLF waves (between 60 and $80 \mathrm{~km}$ ), whereas this parameter of SIC profiles sharply changes from $\sim 0.25$ to $\sim 0.5 \mathrm{~km}^{-1}$ below $72 \mathrm{~km}$. A direct comparison between the VLF results calculated using the WS and SIC-FUV profiles (Figs. 4 and 5) shows that the discrepancy in the simulated VLF signals is caused by the difference in the sharpness parameter.

As evident in Fig. 7(f), the WS profiles derived from the ionospheric fitting are consistent with both previously reported rocket measurements [40] and first-principles calculations [41]; there indeed exists an ionospheric profile with a sharpness parameter close to $0.3 \mathrm{~km}^{-1}$. We also emphasize that the sharp change of $\beta$ value in SIC profiles is not unrealistic [28], but these profiles are not the correct solution to the present VLF problem and the SIC-calculated electron density is likely different from the true ionospheric condition prior to the eclipse.

To quantify the altitude range in which SIC underestimates the electron density, we adopt a "knee"-like ionospheric profile with a "knee" altitude $h_{\mathrm{kn}}[\mathrm{km}]$. This is utilized to imitate the SIC profiles in which the electron density above and below $72 \mathrm{~km}$ can be approximated by two different $\beta$ values. In this "knee" profile, at and above $h_{\mathrm{kn}}, n_{\mathrm{e}}$ is calculated using the WS formula with the $\beta$ value of $0.3 \mathrm{~km}^{-1}$ and different $h^{\prime}$ values (between 70 and $86 \mathrm{~km}$ ). Below $h_{\mathrm{kn}}, \beta$ is chosen to be the same as that of SIC profiles below $\sim 72 \mathrm{~km}\left(0.5 \mathrm{~km}^{-1}\right)$ and $n_{\mathrm{e}}$ is calculated using the electron density at $h_{\mathrm{kn}}\left(n_{\mathrm{kn}}\right)$

$$
n_{\mathrm{e}}(h)=n_{\mathrm{kn}} e^{(0.5-0.15)\left(h-h_{\mathrm{kn}}\right)} \text { for } h<h_{\mathrm{kn}} .
$$

Fig. 8 shows an example of the "knee" profile calculated using a $h^{\prime}$ value of $80 \mathrm{~km}$ and different $h_{\mathrm{kn}}$ values $(55,60$, 65 , and $70 \mathrm{~km}$ ). Defined this way, this "knee" profile is mostly controlled by $h_{\mathrm{kn}}$ and, in this study, we vary $h_{\mathrm{kn}}$ between 50 and $70 \mathrm{~km}$ (step: $1 \mathrm{~km}$ ). If $h_{\mathrm{kn}}$ is sufficiently low that the electron density change below this altitude does not significantly influence VLF propagation, it is conceivable that the "knee" profile becomes equivalent with the WS profile. Thus, the VLF signature would be similar and the "knee" profiles can also explain our VLF measurements. Here, we aim at determining a critical value for $h_{\mathrm{kn}}$ and this value reveals 


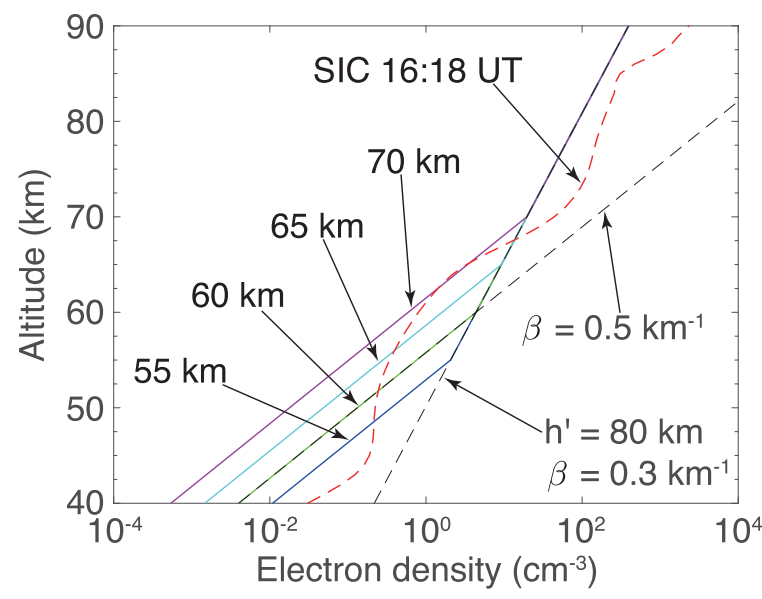

Fig. 8. Example of the "knee" ionospheric profile with $h_{\mathrm{kn}}$ values of 55, 60, 65, and $70 \mathrm{~km}$. Red dashed line: SIC profile at 16:18 UT. Black dashed line with $h^{\prime}=80 \mathrm{~km}$ and $\beta=0.3 \mathrm{~km}^{-1}$ : an example of the best-fit WS profile.

the minimum altitude at which SIC underestimates the electron density. We note that $\beta$ is chosen to be $0.5 \mathrm{~km}^{-1}$ at altitudes below $h_{\mathrm{kn}}$ and only with this assumption we can directly compare the VLF results obtained using the SIC and "knee" profiles. However, VLF propagation is also sensitive to the sharpness parameter below $h_{\mathrm{kn}}$ and future study could take this effect into account.

To this end, we first simulate the VLF propagation using different $h^{\prime}$ and $h_{\mathrm{kn}}$ values. To directly compare between WS and "knee" profiles, we consider that the variation of $h^{\prime}$ during the eclipse $\left[h^{\prime}(t)\right]$ is the same as the best-fitting obtained in the ionospheric fitting [Fig. 7(a)]. For each $h_{\mathrm{kn}}$, the evolution of VLF amplitude and phase in CO and UT can be then obtained using the "knee"-profile-calculated results corresponding to $h^{\prime}(t)$. The comparison with eclipse measurements is shown in Fig. 9. One clearly sees that, as $h_{\mathrm{kn}}$ decreases from 70 to $55 \mathrm{~km}$, the "knee"-profile-calculated VLF results gradually converge to those of WS profiles [see Fig. 5]. The altitude range in which the SIC model underestimates the electron density is between 55 and $72 \mathrm{~km}$. Moreover, these comparison results show that our VLF measurements can be satisfactorily explained as long as the sharpness parameter is $0.3 \mathrm{~km}^{-1}$ above $55 \mathrm{~km}$. This ionospheric profile is close to the rocket measurements reported by Mechtly and Smith [40] [see Fig. 7(f)].

As pointed out by Barabash et al. [41], the sharpness factor at altitudes above $70 \mathrm{~km}$ is most sensitive to variations of $\mathrm{NO}$ concentration, while the concentration of $\mathrm{O}$ and $\mathrm{O}_{3}$ can strongly influence the electron density at altitudes below $70 \mathrm{~km}$. The main reason for the difference between SIC and WS profiles is the default profiles of minor neutral species used in the SIC model, as well as the poorly known negative ion photochemistry [33] and poorly investigated NO abundance. This underestimation can be also caused by either a too large reaction coefficient for negative ion attachment or an inefficient/missing photodetachment process. We note that the latter reason is more likely since more efficient chemical processes can also minimize the time difference between the VLF measurements and modeling results obtained using the SIC profiles (see Fig. 4).

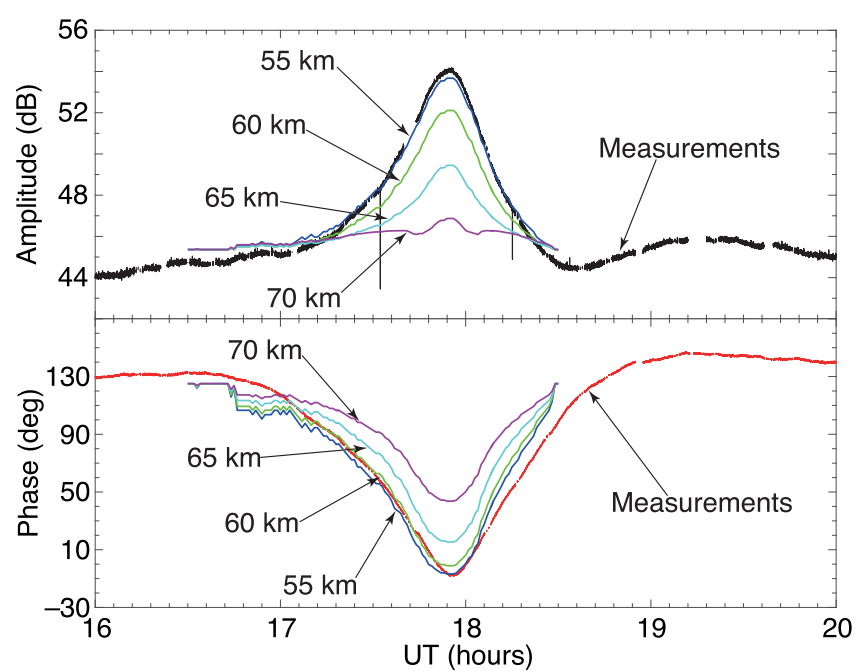

(a)

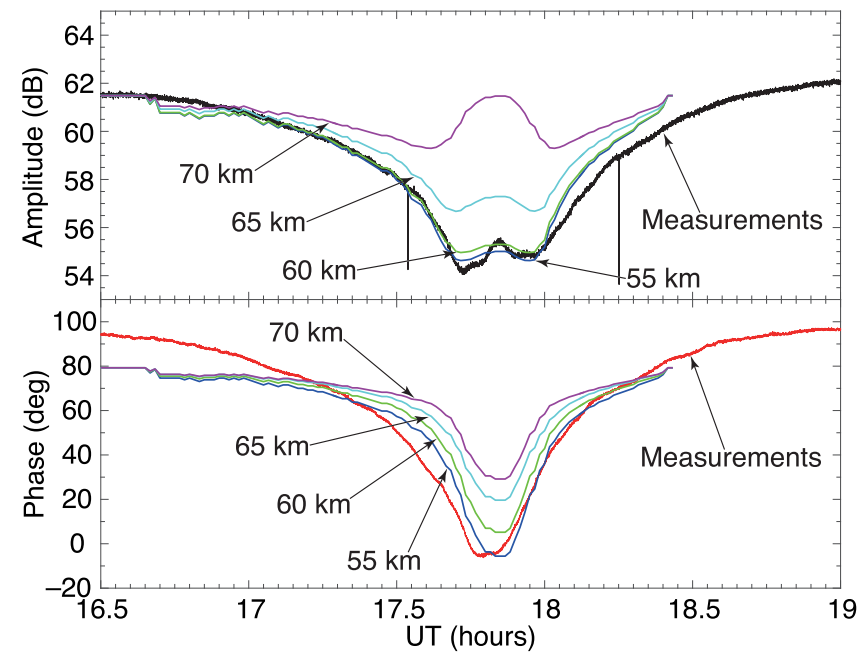

(b)

Fig. 9. (a) Comparison of VLF (Top) amplitude and (Bottom) phase between eclipse measurements in CO and FDTD results obtained using the "knee" profiles with different $h_{\mathrm{kn}}$ values $(55,60,65$, and $70 \mathrm{~km})$. (b) Similar results, but for the VLF measurements in UT.

\section{CONCLUSION AND Discussion}

In this paper, using the NRL solar occultation mask and the SIC model, we have modeled the response of D-region ionosphere to the 2017 total solar eclipse. We have also calculated the VLF signals that would be measured by our receivers during the eclipse using SIC, WS, and "knee" ionospheric profiles. The WS profiles can quantitatively explain the VLF amplitude and phase measurements in both $\mathrm{CO}$ and UT, including the anomalous amplitude enhancement. Moreover, we demonstrate that the set of WS profiles that reconcile satellite and VLF measurements indeed exist as they are consistent with previously reported rocket measurements [40] and first-principles calculations [41].

Because of the default profiles of minor neutral species, the D-region response simulated directly through the SIC modeling using the solar occultation mask is insufficient to reproduce the observed VLF signals. The VLF propagation in the earth-ionosphere waveguide is highly sensitive to the ionospheric density profiles along the path and exhibits a 
complicated response, due to the interference of propagating modes in the waveguide. Doubling or halving the electron density, as well as changing the steepness of density profile at reflection altitudes, can have a pronounced but nonmonotonic effect on the VLF response. As pointed out by Barabash et al. [41], the ionospheric sharpness parameter is closely related to the concentration of minor neutral species in the D-region ionosphere. Therefore, the VLF technique may provide a means to remotely sense the concentration of minor neutral species at and below the effective reflection altitudes of VLF waves.

In the D-region ionosphere, the occultation of solar Lyman- $\alpha$ has the greatest control over the variation of electron density at the altitudes of interests [28]. Our parametric study has found that a reduction in electron density by $\sim 90 \%$ is required to explain the VLF measurements, corresponding to an average occultation of solar Lyman- $\alpha$ by nearly $99 \%$. As clearly shown in Fig. 7(d), our finding of average Lyman- $\alpha$ occultation, as inversely derived from VLF measurements, turns out to be consistent with SDO observation of $\mathrm{He} 30.4-\mathrm{nm}$ emission, as well as previously reported solar measurements that Lyman- $\alpha$ radiation originates mostly from the chromosphere and transition region of the solar atmosphere [32]. The slight difference is likely due to the zero-order approximation and/or solar scattering by the atmosphere/interstellar/heliosphere hydrogen.

Although SIC profiles are different from those best-fitting found in the parametric study, an ionospheric profile such as that calculated by Barabash et al. [41], especially that measured by Mechtly and Smith [40], would be capable of fully explaining our VLF measurements during the 2017 eclipse. However, it is particularly difficult to reproduce this ionospheric profile considering the complexity of chemical reactions included in the SIC model, which is also beyond the scope of this paper. The challenge for future work is to quantify the realistic initial conditions, including profiles of minor neutral constituents and occultation factor of solar Lyman- $\alpha$, which are consistent with not only space-borne measurements but also ground-based VLF observations.

\section{REFERENCES}

[1] D. C. Fritts and M. J. Alexander, "Gravity wave dynamics and effects in the middle atmosphere," Rev. Geophys., vol. 41, no. 1, p. 1003, 2003.

[2] M. V. Codrescu, T. J. Fuller-Rowell, R. G. Roble, and D. S. Evans, "Medium energy particle precipitation influences on the mesosphere and lower thermosphere," J. Geophys. Res., vol. 102, no. A9, pp. 19977-19987, 1997.

[3] S. Solomon, P. J. Crutzen, and R. G. Roble, "Photochemical coupling between the thermosphere and the lower atmosphere: 1. Odd nitrogen from 50 to 120 km," J. Geophys. Res., vol. 87, no. C9, pp. 7206-7220, 1982.

[4] C. E. Randall et al., "Energetic particle precipitation effects on the southern hemisphere stratosphere in 1992-2005," J. Geophys. Res., vol. 112, no. D8, 2007, Art. no. D08308.

[5] R. Barr, D. L. Jones, and C. Rodger, "ELF and VLF radio waves," J. Atmos. Solar Terr. Phys., vol. 62, nos. 17-18, pp. 1689-1718, 2000.

[6] P. Hosseini, M. Gołkowski, and V. Harid, "Remote sensing of radiation belt energetic electrons using lightning triggered upper band chorus," Geophys. Res. Lett., vol. 46, no. 1, pp. 37-47, Jan. 2019.

[7] M. J. Nicolls et al., "Influence of an inertia-gravity wave on mesospheric dynamics: A case study with the poker flat incoherent scatter radar," J. Geophys. Res., vol. 115, Oct. 2010, Art. no. D00N02.
[8] D. McKay-Bukowski et al., "Kaira: The Kilpisjärvi atmospheric imaging receiver array-System overview and first results," IEEE Trans. Geosc. Remote Sens., vol. 53, no. 3, pp. 1440-1451, May 2015.

[9] U. S. Inan, S. A. Cummer, and R. A. Marshall, "A survey of ELF and VLF research on lightning-ionosphere interactions and causative discharges," J. Geophys Res. Space Phys., vol. 115, no. A6, 2010, Art. no. A00E36.

[10] M. Gołkowski et al., "Ionospheric D region remote sensing using ELF Sferic group velocity," Geophys. Res. Lett., vol. 45, no. 23, pp. 12739-12748, 2018.

[11] R. N. Bracewell, "Theory of formation of an ionospheric layer below E layer based on eclipse and solar flare effects at $16 \mathrm{kc} / \mathrm{sec}$," J. Atmos. Terr. Phys., vol. 2, no. 4, pp. 226-235, 1952.

[12] K. Lynn, "The total solar eclipse of 23 October 1976 observed at VLF," J. Atmos. Terr. Phys., vol. 43, no. 12, pp. 1309-1316, 1981.

[13] R. D. Sears, M. G. Heaps, and F. E. Niles, "Modeling the ion chemistry of the D region: A case study based upon the 1966 total solar eclipse," J. Geophys. Res., vol. 86, no. A12, pp. 10073-10086, 1981.

[14] M. A. Clilverd et al., "Total solar eclipse effects on VLF signals: Observations and modeling," Radio Sci., vol. 36, no. 4, pp. 773-788, Jul. 2001.

[15] S. Pal, S. K. Chakrabarti, and S. K. Mondal, " Modeling of subionospheric VLF signal perturbations associated with total solar eclipse, 2009 in Indian subcontinent," Adv. Space Res., vol. 50, no. 2, pp. 196-204, 2012.

[16] T. A. Seliga, "Analysis and results of low-frequency CW rocket propagation experiments in the D region," J. Geophys. Res., vol. 73, no. 21, pp. 6783-6794, Nov. 1968.

[17] J. A. Kane, "D-region electron density measurements during the solar eclipse of May 20, 1966," Planet. Space Sci., vol. 17, no. 4, pp. 609-616, Apr. 1969

[18] M. Jespersen and B. M. Pedersen, "Ionospheric observations during the annular solar eclipse of 20 May 1966-III Four D-region electron density profiles measured by rocket techniques," J. Atmos. Terr. Phys., vol. 32, no. 12, pp. 1859-1863, Dec. 1970.

[19] L. G. Smith, M. K. McInerney, and H. D. Voss, "Electron density and energetic particle precipitation observed during the eclipse of 26 February 1979," J. Atmos. Terr. Phys., vol. 45, no. 7, pp. 427-436, Jul. 1983.

[20] M. B. Cohen, U. S. Inan, and E. W. Paschal, "Sensitive broadband ELF/VLF radio reception with the AWESOME instrument," IEEE Trans. Geosc. Remote Sens., vol. 48, no. 1, pp. 3-17, Jan. 2010.

[21] P. Hosseini, M. Golkowski, H. T. Chorsi, S. D. Gedney, and R. C. Moore, "Using eccentricity to locate ionospheric exit points of magnetospheric whistler mode waves," IEEE Trans. Geosc. Remote Sens., vol. 56, no. 12, pp. 7049-7061, Dec. 2018.

[22] J. D. Huba and D. Drob, "SAMI3 prediction of the impact of the 21 August 2017 total solar eclipse on the ionosphere/plasmasphere system," Geophys. Res. Lett., vol. 44, no. 12, pp. 5928-5935, 2017.

[23] B. J. Harding, D. P. Drob, R. A. Buriti, and J. J. Makela, "Nightside detection of a large-scale thermospheric wave generated by a solar eclipse," Geophys. Res. Lett., vol. 45, no. 8, pp. 3366-3373, 2018.

[24] S. Mrak, J. Semeter, D. Drob, and J. D. Huba, "Direct EUV/X-Ray modulation of the ionosphere during the August 2017 total solar eclipse," Geophys. Res. Lett., vol. 45, no. 9, pp. 3820-3828, 2018.

[25] R. T. Marriott, D. E. St John, R. M. Thorne, S. V. Venkateswaran, and P. Mahadevan, "Ionospheric effects of two recent solar eclipses," J. Atmos. Terr. Phys., vol. 34, no. 4, pp. 695-712, Apr. 1972.

[26] J. R. Lemen et al., "The atmospheric imaging assembly (AIA) on the solar dynamics observatory (SDO)," Solar Phys., vol. 275, nos. 1-2, pp. 17-40, Jan. 2012.

[27] G. Kaplan, J. Bartlett, A. Monet, J. Bangert, and W. Puatua, User's Guide to NOVAS Version F3. 1. Washington, DC, USA: USNO, 2011.

[28] G. P. Brasseur and S. Solomon, Aeronomy of the Middle Atmosphere Chemistry and Physics of the Stratosphere and Mesosphere, 3rd ed. Dordrecht, The Netherlands: Springer, 2006.

[29] J. R. Wait and K. P. Spies, "Characteristics of the earth-ionosphere waveguide for VLF radio waves," Nat. Bureau Standards, Boulder, CO, USA, Tech. Rep. 300, 1964.

[30] E. Turunen, H. Matveinen, J. Tolvanen, and H. Ranta, "D-region ion chemistry model," in STEP Handbook Ionospheric Models, R. W. Schunk, Ed. Boulder, CO, USA: SCOSTEP Secretariat, 1996, pp. 1-25.

[31] R. A. Marshall, "An improved model of the lightning electromagnetic field interaction with the D-region ionosphere," J. Geophys. Res. Space Phys., vol. 117, May 2012, Art. no. A03316. 
[32] T. N. Woods, W. K. Tobiska, G. J. Rottman, and J. R. Worden, "Improved solar Lyman $\alpha$ irradiance modeling from 1947 through 1999 based on UARS observations," J. Geophys. Res. Space Phys., vol. 105, no. A12, pp. 27195-27215, 2000.

[33] P. T. Verronen, M. E. Andersson, D. R. Marsh, T. Kovács, and J. M. C. Plane, "WACCM-D-whole atmosphere community climate model with D-region ion chemistry," J. Adv. Model. Earth Syst., vol. 8, pp. 954-975, Jun. 2016.

[34] W. Xu, R. A. Marshall, X. Fang, E. Turunen, and A. Kero, "On the effects of bremsstrahlung radiation during energetic electron precipitation," Geophys. Res. Lett., vol. 45, no. 2, pp. 1167-1176, 2018.

[35] W. K. Tobiska and S. D. Bouwer, "New developments in SOLAR2000 for space research and operations," Adv. Space Res., vol. 37, no. 2, pp. 347-358, 2006

[36] P. T. Verronen et al., "Diurnal variation of ozone depletion during the October-November 2003 solar proton events," J. Geophys. Res., vol. 110 Aug. 2005, Art. no. A09S32.

[37] R. A. Marshall, T. Wallace, and M. Turbe, "Finite-difference modeling of very-low-frequency propagation in the earth-ionosphere waveguide," IEEE Trans. Antennas Propagat., vol. 65, no. 15, pp. 7185-7197, Dec. 2017.

[38] J. A. Ferguson, "Computer programs for assessment of long-wavelength radio communications, version 2.0: User's guide and source files," Space Naval Warfare System Center, San Diego, CA, USA, Tech. Rep. TD-3030, 1998

[39] S. A. Cummer, U. S. Inan, and T. F. Bell, "Ionospheric D region remote sensing using VLF radio atmospherics," Radio Sci., vol. 33, no. 6, pp. 1781-1792, 1998.

[40] E. A. Mechtly and L. G. Smith, "Seasonal variation of the lower ionosphere at Wallops Island during the IQSY," J. Atmos. Terr. Phys., vol. 30, no. 8, pp. 1555-1561, 1968.

[41] V. Barabash, A. Osepian, P. Dalin, and S. Kirkwood, "Electron density profiles in the quiet lower ionosphere based on the results of modeling and experimental data," Ann. Geophys., vol. 30, pp. 1345-1360, Aug. 2012.

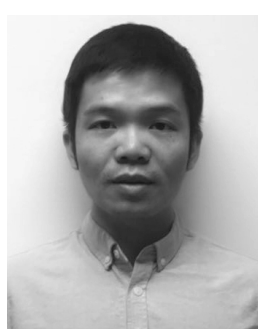

Wei $\mathrm{Xu}$ received the B.S. degree in applied physics from the University of Electronic Science and Technology of China, Chengdu, China, in 2010, and the $\mathrm{Ph} . \mathrm{D}$. degree in electrical engineering from The Pennsylvania State University, University Park, PA, USA, in 2015.

$\mathrm{He}$ is currently a Research Associate with the Department of Aerospace Engineering Sciences, University of Colorado Boulder, Boulder, CO, USA His research interests include computational plasma physics and electromagnetics, energetic radiation from lightning and thunderstorms, radiation belt precipitation and atmospheric effects, atmospheric and space electricity, and numerical modeling and applications in space physics.

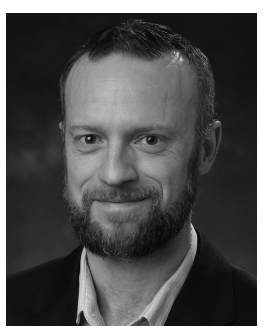

Robert A. Marshall (SM'07-M'15) received the B.S. degree in electrical engineering from the University of Southern California, Los Angeles, CA, USA, in 2002, and the M.S. and Ph.D. degrees in electrical engineering from Stanford University, Stanford, CA, USA, in 2004 and 2009, respectively.

He held various research positions with the Center for Space Physics, Boston University, Boston, MA, USA, and the Department of Aeronautics and Astronautics, Stanford University. He is currently an Assistant Professor with the Ann and H. J. Smead Department of Aerospace Engineering Sciences, University of Colorado Boulder, Boulder, CO, USA. He has authored the book Numerical Electromagnetics: The FDTD Method (Cambridge University Press, 2011) with U.S. Inan. His research interests include the study of lightning-ionosphere interactions, radiation belt precipitation and atmospheric effects, numerical modeling and applications in space physics, and small satellite instrument development.

Dr. Marshall was a recipient of the First Place in the 2007 International Radio Science Union (URSI) Student Paper Competition and the URSI Young Scientist Award in 2011.

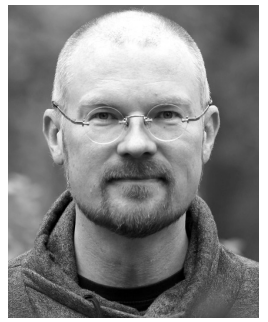

Antti Kero is an Expert in the field of D-region ionosphere studies by remote sensing radio methods and modeling. He is experienced in the EISCAT radar and $\mathrm{HF}$ heating experiments studying the D-region ion chemistry effects.

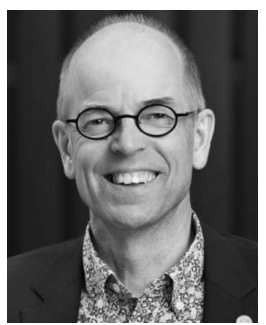

Esa Turunen was the Director of EISCAT Scientific Association from 2009 to 2012, which operates high-power incoherent scatter radars in Svalbard and Northern Scandinavia for upper atmospheric and near-earth space research. He is currently the Director of the Sodankylä Geophysical Observatory (SGO), University of Oulu, Oulu, Finland. He was responsible for ionospheric research and measurements at SGO, coordinating EISCAT radar and rocket measurement campaigns and participating in several EU projects, including mobility schemes and EU COST actions. He is the PI of Finland's participation in the EISCAT_3-D project, where a new 3-dimensionally imaging radar with 30000 individual antennas will be constructed in Northern Finland, Sweden and Norway by 2022. He is the Original Developer of an internationally established theoretical model of the lower ionosphere, the Sodankylä Ion Chemistry model. His research interests include high-latitude ionospheric D region and atmospheric and geospace interactions, including the chemical effect of excess ionization in the middle and upper atmosphere at high latitudes.

Douglas Drob is currently a Research Physicist with the Space Science Division, U.S. Naval Research Laboratory, Washington, DC, USA.

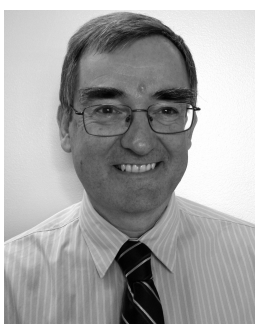

Jan Sojka was born in Duns, U.K. in 1950. He received the B.S. degree in natural philosophy from The University of Edinburgh, Edinburgh, U.K., in 1972, and the Ph.D. degree in space physics from University College London, London, U.K, in 1976.

$\mathrm{He}$ attended Galashiels Academy (GA) Galashiels, U.K. His doctoral and post-doctoral works were carried out at the Mullard Space Science Laboratory, Dorking, U.K., which involved the study of the aurora from observations made by sounding rockets and magnetospheric plasma phenomenon from the European Space Agency's GEOS-I and GEOS-II Satellites. He has chaired several NSF Discipline Working Groups and NASA STDT Teams, is a Trustee of the Utah NASA Space Grant Consortium, and was a Dean of the Heliophysics Summer School, Boulder, CO, USA. Since 1978, he has been engaged in computational modeling and theory of the ionosphere with the Center for Atmospheric and Space Sciences, Utah State University, Logan, UT, USA, where he is also a Professor of physics and the Head of the Physics Department. He is also a Part Owner of Space Environment Corporation, Logan, UT, USA, an applications-oriented space weather corporation. He has authored over 250 scientific papers, given over 450 talks, and coedited a book on Heliophysics.

Dr. Sojka was a recipient of the Oliver Medal for Science (GA), the D. Wynne Thorne Research Award (USU), and the Utah Carnegie Professor of the Year Award for excellence in teaching.

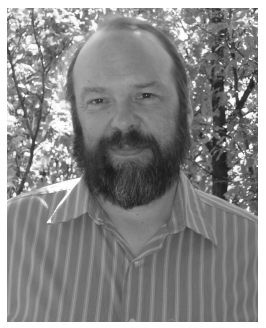

Don Rice received the dual B.S. degree in electrical engineering and in mathematics and the dual M.S degree in electrical engineering and in space physics from University of Alaska Fairbanks, Fairbanks, AK, USA, in 1984 and 1989, respectively.

He currently maintains space weather instrumentation for the Physics Department, Utah State University, Logan, UT, USA, where he teaches upper division laboratory classes. He is involved in ionospheric modeling and radio propagation research projects. 